\title{
INFLUENCE OF MOUNT-FOOT PENETRATION DEPTH ON THE QUANTIFICATION OF DRYING STRAIN\&
}

James Fuller ${ }^{l}$

\begin{abstract}
The need for a drying stress indicator has previously been stated with some research in the past to this means. Given the recent successes in achieving this goal through monitoring shrinkage, the refinement and further understanding of the process was needed, specifically, the influence that penetration of the mount-foot had on the shrinkage data. Four different LVDTmounts were investigated in an attempt to acquire this information.

The results indicated that the depth of penetration influenced the absolute value of shrinkage measured but did not influence determination of when peak stress occurred. The mounts mounted on the face of the board displayed the most stable and informative results.
\end{abstract}

Keywords: drying stresses, kiln control, mount penetration, shrinkage, peak stress, stress reversal

\section{INTRODUCTION}

In 1950, the need for a "tension set detector", a drying stress indicator, for use in the drying of lumber was recommended (Reitz 1950). The first attempt was the recording of the degree of edge barreling (Hill 1986). Edge barreling is the result from shear strain created by the differential drying stresses. Initially, the edge is convex then as the drying stresses reverse the edge becomes concave. The results of the initial attempt were not reproducible because of the inherent mechanical limitations of the mount. Later, simultaneous independent attempts were undertaken to determine when the average moisture content reached Fiber Saturation Point (FSP) by measuring surface strain (Perré et al. 1994; Fortin et al. 1994). Since FSP is a point occurrence, occurring at cellular level, these studies were actually recording some form of stress level. Fuller (1996 and 1999) was able to display stress changes during drying, including both Stress Reversal and Peak Stress in both red oak and sugar maple. Fuller (2000) also successful used the same technique in a commercial run. Northway (2000) recorded removed-discrete measurements of "core diameters", "flying wing" and board width while the MC gradient was obtained for eucalyptus. However, Northway did not extend this into a commercial system.

Work to understand theoretical aspects of drying was performed concurrently with the above laboratory work. This work involved numerous attempts have tried to predict drying stresses using moisture loss and mechanical property calculations. One of the first attempts (Youngs and Bendtsen 1964) showed that the greatest tension stresses occurred at or very near the surface of the board. The mount that was used in the current work to successfully predict stress changes penetrated $7 \mathrm{~mm}$ into the board, deeper into the board than the depth at which Youngs' work indicated the greatest tension stress would be. In the above laboratory work stress changes were determined by the strain release analysis method, as described in Fuller (1995). In performing the strain release analysis method the cross-section of the board is sliced into ten thin slices through the thickness. The limitation of this method is that the slices can only be sliced off the cross-section in a finite thickness and each of these

\footnotetext{
${ }^{1}$ Fuller Drying Labs, P.O. Box 11342, Blacksburg,VA 24062. jfdrywood@earthlink.net

Corresponding author: ifdrywood@earthlink.net

Received: July 27, 2005. Accepted: October 15, 2005.
} 
slices was thicker than the depth at which Youngs predicted the greatest stress was to occur by numerical methods. However, considering the limitation of slice thickness, the new method (Fuller 1996) was successful.

Given the success of the new method, it is of interest to determine what influence the penetration depth of the mount-foot has on the display of stress. This is the object of the present study.

\section{MATERIAL AND METHODS}

Two runs using one sample each were performed in a chamber that was maintained at $44^{\circ} \mathrm{C}$ drybulb and $41^{\circ} \mathrm{C}$ wet-bulb. The initial and final weights of the sample were recorded. A Moisture Content (MC) section was cut after the testing to determine the average weight loss rate. The samples were $32 \mathrm{~mm}$ thick by $150 \mathrm{~mm}$ wide by $340 \mathrm{~mm}$ long red oak which had the ends sealed with aluminum paint. This study was not concerned with material variability nor replicating the variability of a kiln because these issues were addressed in previous papers (Fuller 1999 and 2000).

Four Linear Variable Displacement Transducers (LVDT) were mounted in the same four positions for each of the two runs (Figure 1). Three positions were picked to display the influence of the mountfoot penetration and the fourth was to determine if thickness strain was a helpful parameter to monitor. The first was mounted on brackets that were attached to the center of the edge of the board to measure the shrinkage with the equivalence of $50 \%(16 \mathrm{~mm})$ penetration. The second had the same penetration as the original study (Fuller 1999), with the bracket foot penetrating $7 \mathrm{~mm}$ into the board; this represented approximately $22 \%$ thickness penetration. The third had zero penetration with the mount having a knurled under-side placed on the surface which was held in place by a spring. A steel ball was placed between the spring and mount to insure the mount was independent of the spring. The fourth was a pliers-like mount for recording thickness change. This mount was centered approximately $20 \mathrm{~mm}$ from the edge. All four were mounted as close to each other as possible within the physical limitations of the mounts. The distances between the brackets of each sensor were recorded after each run and were properly accounted for in the standard shrinkage calculations. The LVDT's were connected to a computer which recorded the electrical signals from the four LVDT's every 15 minutes. These signals were then converted to shrinkage values.

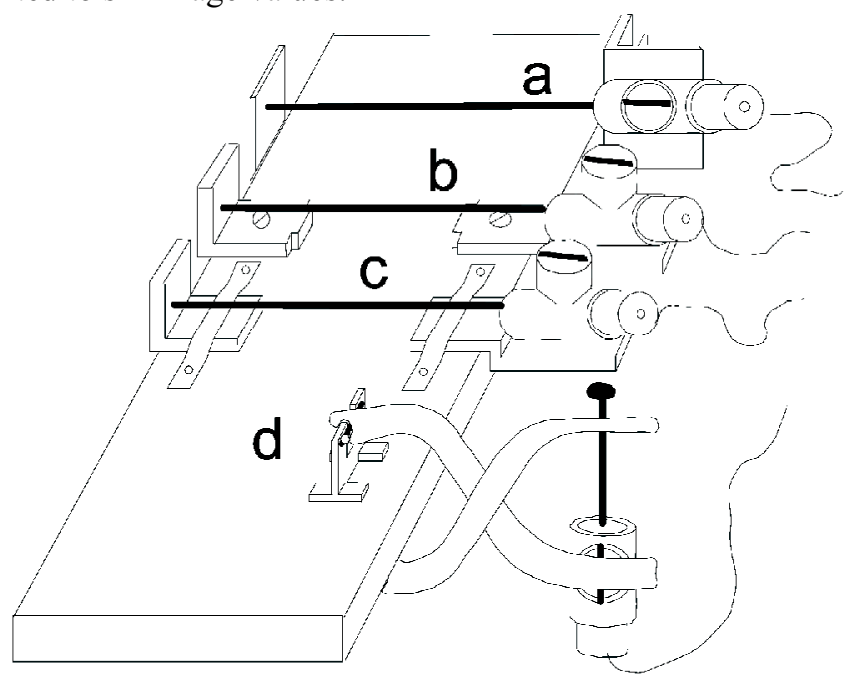

Figure 1. The arrangements of the four mounts are shown: a) penetration of 50\% thickness, total width measurement with the brackets screwed at the center of the edge, b) penetration of $7 \mathrm{~mm}$ with mount screwed to the surface, c) zero penetration with amount that had a knurled under-surface held in place by a spring on a ball bearing, and d) thickness measure by a "pliers" type mount. 


\section{RESULTS AND DISCUSSION}

The durations of the runs were 15 and 12 days respectively for runs one and two. Both runs were discontinued at these respective times because of a malfunction of the chamber controls which resulted in uncontrolled moisture sorption. After the discontinuation of the runs, cross sections were cut from the samples and oven-dried at $105^{\circ} \mathrm{C}$ for $\mathrm{MC}$ determination. From the $\mathrm{MC}$ values the average moisture loss rates were calculated and found to be 3.4 and $3.8 \%$ /day respectively. These values include the moisture that was gained between when the chamber malfunctioned and discontinuation of the run. Therefore, the measured MC loss rates are lower than actual rates.

Both runs produced similar shrinkage results. In the second run the samples were isolated from vibrations better giving more stable LVDT readings, therefore only the results from the second run are displayed and discussed.

Figure 2 displays the shrinkage of the four mounts. The data for the $50 \%$ penetration mount was very erratic. The erratic nature was from the inherent mechanical weaknesses of this mount, similar to Hill's problems (1986). Good contact with the edge was not maintained because the screw projected out of the edge more as the wood shrank. Also, the barreling of the edge did not provide a firm substrate. The results from the thickness mount also displayed some variability. The reason for this variability in the thickness mount was its inherent mechanical design resulting in sensitivity to any vibration or movement that the sample was exposed to. Although the data from the thickness mount displayed variability, the data was reproducible and of sufficient quality to obtain reliable trends. The results from this mount, theoretically, should be influenced by the relative distance from the edge of the board. Near the edge, the mount may be recording shrinkage of wood that had a low moisture gradient. In contrast, if the mount was positioned far from the edge, it would be recording shrinkage of wood that contained a large non-uniform moisture gradient. Due to limited resources and because the main emphasis of this study was penetration depth, only one thickness position was monitored. The surface and $7 \mathrm{~mm}$ mounts displayed swelling after day 11 (Figure2). This was due to the chamber malfunctioning and the wood gaining moisture.

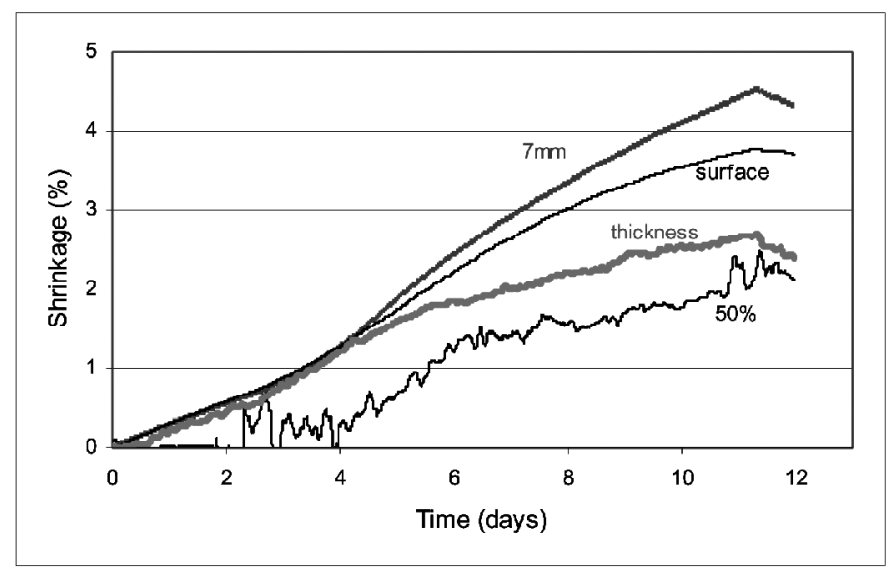

Figure 2. Shrinkages of the four mounts labeled: surface, $7 \mathrm{~mm}, 50 \%$ and thickness.

The data for the surface, $7 \mathrm{~mm}$ and thickness mounts displayed uniform shrinkage rates for approximately three days, then the rate increased which indicated a stress change. The rates subsequently then reduced gradually. Figure 3 displays an enlargement of the surface and $7 \mathrm{~mm}$ shrinkage curves. It can be seen from Figure 3 that the surface mount initially recorded greater shrinkage than the $7 \mathrm{~mm}$ 
mount. After the stress change, the $7 \mathrm{~mm}$ mount recorded a greater increase shrinkage rate than the surface mount. This follows the theoretical drying stress development and how it would predict the display by each mount. As each layer, through the thickness of the board, responses to the transfer of water out of that layer, it acts to produce a mechanical action of shrinkage strain. The other layers, in response, react against the shrinking layers. The surface begins to dry first with subsequent internal layers starting to dry later and slower. Once the surface approaches equilibrium with the atmosphere, the moisture loss rate of this layer reduces, the shrinkage rate reduces and thereby the stress level reduces. Layers further in would first react to the surface layer. When these subsequent layers start to lose moisture they act by shrinking and reducing the stress level. With it not resisting the surface, the net shrinkage rate would increase with the shrinkage rate of this layer being greater than the surface layer. The surface layer shrinkage will ultimately be surpassed by the inner layers. Each mount will display a sequence of actions and reactions by layers able to influence the mount. This is reflected by the recorded shrinkage rates by the mounts.

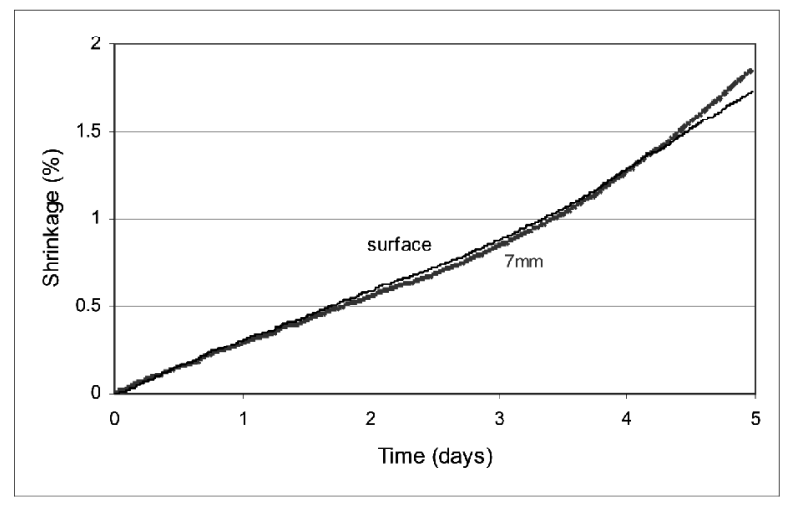

Figure 3. Shrinkage of two mounts (surface and $7 \mathrm{~mm}$ ) displaying how the surface shrinkage rate increases less than the $7 \mathrm{~mm}$ shrinkage rate after the stress changes.

To accurately determine when the shrinkage rate changed, two methods of analysis were used: Total/Running Slope method (TRS) and Shrinkage Difference method (SD). The total/running slope method was used by Fuller (1999) for determining stress. This method compares the total slope of the shrinkage curve with the running slope. The SD method involves subtracting the $7 \mathrm{~mm}$ shrinkage from the surface shrinkage. Figure 4 displays results of both methods from the surface mount.
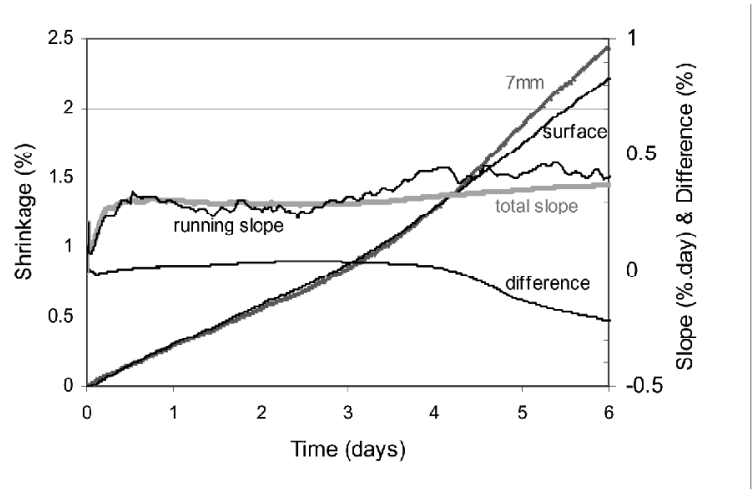

Figure 4. Shrinkage of the surface and the three curves used to analyze when the stress change. 
The concept underlying the TRS method is that when the slope changes, the running slope should respond quicker than the total slope. (Each of these values are described fully in Fuller 1999.) When peak stress occurs and the shrinkage slope increases, the running slope will become greater than the total slope, as occurred in Figure 4 at 2.75 days.

The concept underlying the SD method is that as the slope in a curve, that initially had a smaller slope, increases faster than a curve that initially had a greater slope, the distance between the two would reach a maximum at the point of slope changes. After the difference reaches a maximum, it would subsequently decrease. Figure 4 displays this occurring at 2.6 days.

The $7 \mathrm{~mm}$ and thickness mounts are similar and therefore are not be displayed. The transition times calculated for all four LVDT's are listed in Table 1. All three stable mounts produced different absolute shrinkage values. However, they produced similar curves and indicated that the stress change occurred at approximately the same time. In contrast, the difference between the surface and $7 \mathrm{~mm}$ mounts, indicated that the stress change occurred 3.6 hours sooner. As previously noted, the $50 \%$ penetration mount gave erratic values and therefore it was impossible to accurately determine the time that it indicated stress change, but the shrinkage increased at about the same time as the others (Figure 2). The values for thickness mount should not be used to indicate when stress change occurs until further study is performed because of the position, as mentioned earlier, this is theoretically very critical to indicating stress change.

In developing a commercial piece of equipment, reliability of the LVDT signal, ease of use and complexity of the instrument needs to be considered. At this point, the $50 \%$ penetration and thickness mounts do not produce a reliable signal so they should not be used. Use of the SD method of analysis requires two mounts and therefore is both more complex and difficult to use than a single mount. The added difficulty of the SD method may well be offset by the ability of the SD method to indicate that stress change has occurred slightly earlier than a single surface or deeper penetration mount. On this basis further consideration of the SD may be warranted.

Table 1. Times when stress change was indicated to occur by each method and mount for each run.

\begin{tabular}{|l|c|cc|}
\hline \multirow{2}{*}{ Mount } & \multirow{2}{*}{$\begin{array}{c}\text { Analysis } \\
\text { Method }\end{array}$} & \multicolumn{2}{c|}{ Time (days) } \\
\cline { 3 - 4 } & & Run 1 & Run 2 \\
\hline Surface \& 7mm & Difference & 3.2 & 2.6 \\
Surface & Total/Running & 2.5 & 2.75 \\
$7 \mathrm{~mm} 22 \%$ & Total/Running & 2.2 & 2.75 \\
$50 \%$ & Total/Running & 3 & $2.8-2.95$ \\
Thickness & Observational & 2.9 & 2.77 \\
\hline
\end{tabular}




\section{CONCLUSIONS}

Previously, the material response-based method used to monitor and control a dry kiln had been shown to be an effective method to increase efficiency of lumber drying while at the same time improve the quality of the resulting lumber. The need to determine what influence the mount-foot penetration had on the measurement of the shrinkage and what it indicates was recognized. In this study it was shown that just as the theory of drying stress development would predict, shrinkage at different depths would result in different values. It was also shown that while the penetration influenced the absolute shrinkage values it did not significantly influence when stress change was indicated. The time difference was approximately 3 hours. This work demonstrates that from the equipment designers point of view there is some latitude available in choosing the penetration of mounts and the method of analysis. There may still be possibilities in using a 50\% penetration or "pliers" mount if the design is improved in some way to overcome the inherent weaknesses in the present design. Further work in this area may prove fruitful.

\section{NOTE}

\$SUS Patent: 5,992,047

\section{REFERENCES}

Fortin, Y.; Ilieva, M.; Cloutier, A.; Laforest, P. 1994. Potential use of a semi-ring extensiometer for continuous wood surface strain measurement during kiln drying. 4th IUFRO International Wood Drying Conference. August 9-13, Rotorua, New Zealand. A. Haslett and F. Laytner, Compilers. p. 329334.

Fuller, J. 1995.Conditioning Stress Development and Factors That Influence the Prong Test. Res.Pap. FPL-Rp-537. Madison, WI: US. Department of Agriculture, Forest Service, Forest Products laboratory.6p.

Fuller, J. 1996. Kiln control based on changing shrinkage rate. US patent 5,873,182

Fuller, J. 1999. Determining the source of changing shrinkage rates during drying. Drying Technology, 18(1):261-278.

Fuller, J. 2000. Additional sources of changing shrinkage rates during kiln drying. Drying Technology. 18(4\&5):1023-1032.

Hill, J.; Lessard, R.1986. Automated kiln drying systems of the third kind. Proc. 47345 Eastern Hardwoods: The resource, the industry and the markets. For. Prod Society: 104-114.

Northway, R. 2002. Techniques to monitor drying stresses and dimensional changes in timber from plantation-grown eucalyptus for kiln schedule development and kiln control. Maderas. Ciencia y tecnologia 4(2):148-154.

Perre, P.; Canteri, L.; Martin, M. 1994. Surface strain measurements during convective drying at high temperature as a quality criterion. 4th IUFRO International Wood Drying Conference. August 9-13 Rotorua, New Zealand. A. Haslett and F. Laytner, Compilers. p. 465-466. 30.

Rietz, R. 1970. Accelerating the kiln-drying of hardwoods. Southern Lumberman 221(2741): 19-

Youngs,R.; Bendtsen, B.1964. Tensile, compressive \& shearing stresses developed in red oak as it dries. Forest Products Journal 14(3):113-118. 